\title{
Utilisation des drêches de brasserie desséchées dans l'alimentation du poulet de chair en régions tropicales
}

\author{
par R. BRANCKAERT*
}

\begin{abstract}
RÉSUMÉ
L'auteur, dans le but de préciser les conditions d'utilisation pour l'alimentation du poulet de chair des drêches de brasserie desséchées produites au Cameroun, a conduit un essai sur 6 lots de 100 poussins qui ont reçu 3 régimes différents à partir du 4e jour. La proportion de drêches de brasserie incorporées dans les rations a été selon les lots de :0 p. 100,20 p. 100 et 40 p. 100 .

Les résultats sont analysés statistiquement. L'efficience du régime à 20 p. 100 de drêches desséchées est supérieure ou lot témoin. Par contre, le lot alimenté avec une ration contenant 40 p. 100 de drêches desséchées a eu un développement ralentı ef même inhibé, cependant que l'on notait une augmentation significative de l'indice de consommation.
\end{abstract}

L'utilisation maximale des ressources locales dans l'alimentation des animaux domestiques est un problème intéressant à la fois le zootechnicien, le nutritioniste ef l'économiste. Ce problème est particulièrement important pour les pays d'Afrique Centrale, contraints d'importer encore la plus grande part ou la part qualitativement la plus importante (concentrés protéiniques, vitamines, oligo-éléments) des alıments composés pour le bétail. Et le fait que l'importation de ces produits s'accompagne du paiement de droits de douane très élevés, n'arrange évidemment pas les choses.

Ainsi, la création récente de plusieurs industries agricoles (huileries et rizeries principalement) a permis de mettre sur le marché local des aliments pour le bétail toute la gamme de leurs sous-produits. Dans ce même ordre d'idées les Brasseries du Cameroun, ont mis en route d̀ Douala, en septembre 1966, une installation de déshydratation de drêches. Cette installa-

(*) Expert F. A. O. en production animale. Université Fédérale du Cameroun, Ecole Fédérale Supérieure d'Agriculture, département de Zootechnie. tion permet de produire annuellement, environ 120 tonnes de drêches desséchées à partir de 650 tonnes de drêches fraîches. Le prix de vente du $\mathrm{kg}$ de drêches desséchées varie de 15 d̀ 18 F CFA * suivant la quantité commandée.

\section{LES DRECHES DE BRASSERIE}

Ce sont les résidus solides que l'on trouve au fond des cuves de brassage après la saccharification de l'amidon du malt, les prodults liquides formant le moût.

Les éléments de base utilisés par les Brasseries du Cameroun pour la fabrication de leurs bières sont le malt, le riz et le mals, ces deux dernières céréales étant présentes à raison de $400 \mathrm{~kg}$ par brassin.

A l'état naturel, les drêches contiennent une très forte proportion d'eau (75 à 80 p. 100). Comme elles sont riches en matières fermentescibles et qu'elles séjournent longtemps aux températures favorables au développement des

(*) 1 F CFA $=0,02$ F Français. 
microorganismes, elles sont susceptibles de se gâter très rapidement en se refroidissant.

Leur composition moyenne est la suivante :

Eau

$75-80$ p. 100

Matières protéiques brutes ......

5 p. 100

Matières grasses ...............

Extractif non azoté .............

Fibre brute .................

2 p. 100

10 p. 100

5 p. 100

1 p. 100

Leurs teneurs en $P_{2} O_{5}$ et en minéraux sont d'autant plus élevées que l'eau de brassage contient plus de calcium et de magnésium.

La drêche fraîche est donc un alıment de durée limitée, devant être utilisé dans les 48 heures, et donc, au voisinage immédiat des brasseries productrices.

II n'en est pas de même de la drêche desséchée : le séchage fait en effet tomber l'humidité au dessous de 10 p. 100 et procure un aliment de haute valeur nutritive, dont la teneur en protéines ascille entre 20 et 30 p. 100.

Voici, d'après MORRISON, la composition bromatolagique de deux échantillons de drêches séchées :

Matières sèches ........... 93

Mat. prot. brutes ......... 27,5

23,5

Mat. prot. digestibles ........ 22

Relation nutritive $\left(^{*}\right) \ldots \ldots \ldots$ 2,1

17

Matières grasses ........... 6,5

Fibre brute ............. 14

Extractif non azoté ........ 41

Minéraux .............. 3,7

15,5

43,5

Calcium ............... 0,30

4,

Phosphore ............ 0,51

0,32

0,54

Unités fourragères $\ldots \ldots \ldots, 0,7$
Extractif non azoté ........ 41,9

Insoluble chlorhydrique.... . 1,9

Calcium .............. 0,516

Phosphore ........... 0,718

(Remarque : toutes les valeurs sont rapportées en p. 100 de la matière sèche).

Les teneurs sont donc assez comparables à celles qui sont indiquées par MORRISON, avec toutefois un taux minéral un peu plus élevé dans les drêches camerounaises. La drêche desséchée constituant ainsi un aliment concentré de bonne valeur, il est curieux qu'à ce jour, peu d'essais concernant son utilisation aient été relatés. II est certain toutefois que l'installation d'un déshydrateur de drêches fraîches est une opération onéreuse, par laquelle seul un petit nombre de brasseries s'est laissé tenter.

II est évident que le Cameroun se doit de profiter au maximum de cette occasion qui lui est offerte de procurer à son cheptel des protéines à bon compte. Cependant, le coût du transport grève assez lourdement le prix de vente du produit, de sorte qu'il n'est possible de l'utiliser que dans les régions proches de Douala. Or, dans le Centre-Sud Camerounals, il existe une aviculture semi-industrielle florissante, dont le développement est l'œuvre de divers organismes officiels ou privés. C'est donc dans ce secteur de l'économie animale qu'il faut promouvoir l'utilisation des drèches de brasserie desséchées. Il était tout indiqué d'entamer au plus tôt des esscis sur les possibilités d'incorporation maximale de la $D$. $D$. dans les alıments composés pour volailles. Comme les résultats sur poules pondeuses réclament au moins un an de relevés, la première expérimentation a été entamée sur poulets de chair.

En 1962-1964, KIENHOLZ ef THORNTON (Université du Colorado - Fort-Collins U. S. A.) ont réalısé des essais semblables ef ont obtenu des résultats assez encourageants. Cependant, les aliments de base qu'ils avalent utilisés (milocorn et tourteau de soja) diffèrent considérablement des mêmes ingredients employés au Cameroun (maïs et tourteau de coton). II était donc intéressant de répéter - vu les problèmes d'intersupplémentation - l'expérimentation dans les conditions locales.

De plus, il est à noter que l'essai sur l'engraissement du poulet de chair est original.
(*) La relation nutritive est le rapport entre la quantité de matières protéiques digestibles ef les autres composés organiques alimentaires (hydrates de carbone et graisses). 


\section{MÉTHODE ET RÉSULTATS}

La présente note a trait à un essai mené sur poulets de chair (souche PETERSON 160), importés de France à l'âge d'un jour, de novembre 1966 à février 1967. Un phénomène curieux a perturbé le début de l'expérience : un pourcentage considérable $(10 \mathrm{p}, 100)$ de sujets sont morts dans les deux premières semaines, sans qu'aucune cause infectieuse ou infestante n'ait été mise en lumière à la faveur d'examens répétés. C'est la seconde fois que nous observons ces mortalités en série et nous l'attribuons au stress intense subi par les poussins provenant d'un pays à température très fraîche, et subissant des transbordements successifs (Parıs-Dovala-Yaoundé), dans des hangars ouverts à tous les vents et dans des cales dont la température moyenne $n^{\prime}$ excède jamais $15^{\circ} \mathrm{C}$.

Le même phénomène s'est produit lors d'une expédition ayant eu lieu au mois de décembre, tandis que, sur des poussins importés en juillet, nous n'avons subi qu'un pourcentage infime $(0,5$ p. 100) de pertes.

Les 600 poussins ont été répartis au hasard par lots de 100 dans 6 poussinnières et soumis à trois régimes différents à partir du $4^{e}$ jour. Pendant les trois premiers jours, ils ont tous reçu de la farine de mais. Les régimes utilisés étaient à base de 0 p. 100 , de 20 p. 100 et 40 p. 100 de drêches desséchées. Le régime témoin à 0 p. 100 avait été déterminé antérieurement par essai sur la même souche de poulets.

TABLEAU $N^{\circ} \mathrm{I}$

Rations utilisëes

\begin{tabular}{|c|c|c|c|c|c|c|}
\hline & \multicolumn{3}{|c|}{ Démarrage $(0-6$ semaines $)$} & \multicolumn{3}{|c|}{ Finition ( 6 semaines-abattage) } \\
\hline & Têmoin & 20p.100D.D. & $40 \mathrm{p} \cdot 100 \mathrm{D} \cdot \mathrm{D} \cdot$ & Témoin & $20 \mathrm{p} \cdot 100 \mathrm{D}, \mathrm{D}$ & 40p.100D.D. \\
\hline Maìs & 68 & 60 & 45 & 80 & 65 & 55 \\
\hline D.D. & 0 & 20 & 40 & 0 & 20 & 40 \\
\hline Tourteau coton & 12 & 10 & 5 & 10 & 5 & 0 \\
\hline Tourteau arachide & 10 & 0 & 0 & 0 & 0 & 0 \\
\hline Concentre (x) & 10 & 10 & 10 & 10 & 10 & 10 \\
\hline
\end{tabular}

(x) le concentré, à base de farines animales, contient également des vitamines, des minêraux, un coccidiostatique et un antibiotique.

TABLEAU $N^{\circ} I I$

Analyse bromatologique de la ration démarrage.

\begin{tabular}{|c|c|c|c|c|c|c|c|}
\hline & & \multicolumn{2}{|c|}{ Témoin o p.100 D.D. } & \multicolumn{2}{|c|}{$20 \mathrm{p} \cdot 100 \mathrm{D}, \mathrm{D}$} & \multicolumn{2}{|c|}{40 P.100 D.D. } \\
\hline & & Lot $n^{\circ} 1$ & Lot $n^{\circ} 2$ & Lot $\mathbf{n}^{\circ} \mathbf{1}$ & Lot $n^{\circ} 2$ & Lot $n^{\circ} 1$ & Lot $\pi^{\circ} 2$ \\
\hline \multirow{9}{*}{ - } & Matière sèche & 86,45 & 87,09 & 86,25 & 86,50 & 87,30 & 87,32 \\
\hline & Matières protēiques brutes & 19,68 & 19,54 & 19,35 & 18,60 & 21,25 & 21,21 \\
\hline & Matières grasses & 4,66 & 5,21 & 3,93 & 4,64 & 4,94 & 5,49 \\
\hline & Cellulose & 6,70 & 7,17 & 4,30 & 4,91 & 7,55 & 7,23 \\
\hline & Extractif non azoté & 50,99 & 50,49 & 54,69 & 54,02 & 48,30 & 48,07 \\
\hline & Matières minérales totales & 4,42 & 4,69 & 3,93 & 4,33 & 5,26 & 5,32 \\
\hline & Calcium & 0,643 & 0,720 & 0,626 & 0,700 & 0,860 & 0,830 \\
\hline & Phosphore & 0,606 & 0,550 & 0,575 & 0,520 & 0,734 & 0,620 \\
\hline & Insoluble Chlorhydrique & 0,84 & 1,04 & 0,49 & 0,52 & 0,91 & 0,82 \\
\hline
\end{tabular}

Remarque : les valeurs sont exprimées en pourcentage du produit brut. 
TABLEAU $N^{\circ}$ XII

Evolution du crô̂t des animaux

\begin{tabular}{|c|c|c|c|}
\hline & $\begin{array}{l}\text { Lot témoin } \\
\text { (moyenne des deux lots) }\end{array}$ & (moyerne des deux lots) & $\begin{array}{l}40 \\
\text { (movenne des deux lots) }\end{array}$ \\
\hline Arrivêe & 39 & 39 & 39 \\
\hline 3 sema1nes & 152 & 171 & 148 \\
\hline 4 semaines & 247 & 228 & 168 \\
\hline 5 semaines & 379 & 379 & 231 \\
\hline 6 semaines & 499 & 488 & 332 \\
\hline 7 semaires & 602 & 692 & 462 \\
\hline B semaines & 935 & 982 & 701 \\
\hline 9 semaines & 1146 & 1158 & 925 \\
\hline 10 semaines & 1180 & 1305 & 1019 \\
\hline 11 semaines & 1409 & 1563 & 1141 \\
\hline 12 semaines & 1496 & 1667 & 1139 \\
\hline 13 semaines & - & - & 1148 \\
\hline
\end{tabular}

TABLEAU $\mathrm{N}^{\circ} \mathrm{IV}$

Indices de consommation

\begin{tabular}{|l|c|c|c|}
\hline & Témoin & $20 \mathrm{p} .100$ D.D. & 40 . 100 D.D. \\
\cline { 2 - 4 } let lot & 2,961 & 2,936 & 3,839 \\
2ème lot & 3,167 & 2,745 & 3,653 \\
Moyenne & 3,055 & 2,897 & 3,744 \\
\hline
\end{tabular}

On notera la teneur assez faible en cellulose de la ration à 20 p. 100 D. D. Les poussins ont été pesés à l'arrivée, ensulte chaque semaine à partir de 3 semaines jusqu'à 13 semaines (tableau 3).

Les indices de consommation des différents lots sont repris dans le tableau 4.

L'interprétation statistique de cette dernière donnée s'avère assez difficile du fait qu'elle représente le rapport de deux variables aléatoires $\frac{(P . T .)}{C . T \text {. }}$.

La variance d'un rappart de deux variables aléatoires $z=\frac{x}{y}$ de distribution normale et de moyenne $\bar{x}$ ef $\bar{y}$, a été calculée en utilısant la formule donnée par LISON dans son ouvrage : Statistique appliquée à la Biologie expérimentale, page 236.

Le nombre de degrés de liberté a été calculé par la formule de WELSH :

(Biométrika 29/1937, pages 350-362).
Tableau 5

Analyse de la variance des indices de consommation. Lot témoin

$$
\begin{aligned}
& \bar{z} 1=3,06 \\
& \text { variance }=0,000881277 \\
& \text { nombre de ddl }=1,4 .
\end{aligned}
$$

Lot à 20 p. 100 D. D.

$$
\begin{aligned}
& \bar{z} 2=2,90 \\
& \text { Variance }=0,00281482 \\
& \text { nombre de } d d l=1,07
\end{aligned}
$$

Lot à 40 p. 100 D. D.

$$
\begin{aligned}
& z 3=3,75 \\
& \text { Variance }=0,02782681 \\
& \text { Nombre de ddl=1,01. }
\end{aligned}
$$

a) Variance de la différence entre les indices de consommation des lots témoin et des lots à 20 p. $100 \mathrm{D}$. D.

$t=3,663$ pour 2,47 degrés de liberté. Différence non significative.

b) Variance de la différence entre les indices de consommation des lots témoins et des lots à 40 p. $100 \mathrm{D}$. D. $\dagger=4,07$ pour 2,41 degrés de liberté. Différence significative cu seuil de 5 p. 100. 
c) Variance de la différence entre les indices de consommation des lots à 20 p. 100 D. D. et 40 p. 100 D. D. $t=5,2$ pour 2,08 degrés de liberté. Différence significative au seuil de 5 p. 100.

\section{COMMENTAIRES}

1. Aucune différence n'est perçue dans la comparaison des croissances des sujets des lots témoin et des sujets soumis au régime à 20 p. 100 D. D. Au contraire, tout au long de la croissance, les sujets soumis au régime 20 p. 100 D. D. ont accusé un léger gain de poids qui, à 12 semaines, s'est même élevé à 11,43 p. 100.

2. De même, aucune différence significative n'a pô être dégagée de l'analyse de variance des indices de consommation des lots soumıs aux régimes témoin, et à 20 p. 100 D. D.

3. Par contre, les sujets soumis au régime à 40 p. 100 D. D. ont accusé un retard considérable de croissance, et celle-ci s'est même pratiquement arrêtée à l'âge de 11 semaines et au poids de $1.140 \mathrm{~g}$. En effet, dans les deux semaines suivantes, les poulets n'ont plus réalisé que $7 \mathrm{~g}$ de gain de poids.

De même, l'analyse de la variance des indices de consommation de ces sujets comparés à ceux soumis aux autres régimes relève une différence significative, qui, en pratique, se chiffre par une consommation accrue de $700 \mathrm{~g}$ d'aliment environ par kilo de poids vif.

\section{CONCLUSIONS}

1. L'incorporation de drèches desséchées dans la ration du poulet de chair, à raison de 20 p. 100 , n'entraîne aucun retard de crolssance par rapport à un régime classıque.

Il semble même que l'efficience alimentaire d'un régime à 20 p. 100 D. D. soit un peu supérieur à celle du régime témoin.

2. L'incorporation de taux plus élevés de drèches desséchées - en particulier celui de 40 p. 100 - entraîne un ralentissement et même une inhibition du développement des sujets, et une augmentation significative de l'indice de consommation.

\section{REMERCIEMENTS}

Les laboratorres de Nutrition de l'I. E. M. V. T. à Maisons-Alfort (Dr RIVIËRE) et de l'I. R. C. A.M. Yaoundé ( $M$. FAVIER) ont procédé aux analyses bromatologiques.

M. LOTODE, statisticien à I'Institut Français du Café et du Cacao, a prêté son concours dans l'interprétation statistique des résultats.

Qu'ils en soient sincèrement remerciés.

\title{
SUMMARY
}

\section{The use of dried Brewer's grains in the food of broiler in tropical regions}

\begin{abstract}
Experiment has been carried oul on 6 groups of 100 chicks, which were given 3 different diets from the 4th day, in order to study the utilization of the dried brewer's grains in the food of meat producing chicken. The percentage of brewer's grains in the diet was respectively in the 3 groups :0 p. 100, 20 p. 100, and $40 \mathrm{p}, 100$.

The results have been slatisticaily analysed. The results recorded with the 20 p. 100 diet were better than those oblained in the check group. The group fed with a diet containing 40 p. 100 of dried brewer's graıns showed a slower and even inhibited growth rate while a significant increase of the consumption rate was recorded.
\end{abstract}

\section{RESUMEN}

Urilización de las heces de cerveceria desecadas en la alimentación de la gallina para carne en regiones tropicales

Este estudio tiene por objeto delerminar las condiciones de utilización de las heces de cerveceria desecadas, praducidas en el Comerún, para la alimentación de la gallina para carne. Se alımentaron 6 lotes de 100 pollitos con 3 raciones 
diferentes a partir del $4^{a}$ dia. El termino medio de las heces de cerveceria incorporadas en las raciones llegó, según los lates, a : 0 p. 100, 20 p. 100 y $40 \mathrm{p} .100$.

Se analizaron los resultados estadisticamente.

La eficacia de la ración con 20 p. 100 de heces de cerveceria es superiora a la de la ración testigo. En cambio, el loie alimentado con una ración conteniendo 40 p. 100 de heces desecadas mostró un crecimiento despacioso $y$ hasta inhibido, mientras se notaba un aumento significativo del indicia de consumo.

\section{BIBLIOGRAPHIE}

MORRISON (F. B.). - Feed and feeding. 22nd ed. Clinton, The Morrison Publishing Co., 1959.

PICCION! (M.). - Dictionnaire des Aliments pour les animaux $3^{e}$ édit. Mise à jour et adaptation par J. HARDOUIN, Bologna, Edagricole, 1965.

MONGODIN (B.) et RIVIERE (R.). - Analyse bromatologique de 150 aliments de l'Ouesł Africain. Rev. Elev. Méd. vét. Pays trop., 1965,18 (2) : 183-218.

PURY (P. de) - Comment élever les poules. Guide d'Aviculture équatoriale. Yaoundé, Centre de littérature évangélique, 1966.

- L'alimentation des volailies dans les pays tropicaux et subtropicaux. Rome, O. A. A., 1965 (Coll., Progrès et mise en valeur. Agriculture, $n^{\circ} 82$ ).
KIENHOLZ (E. W.). - Brewer's dried grains as a protein supplement in chicken starter, grower, layer and breeder dieis. Feedstuffs, 1964, $36(20): 34$.

THORNTON (P. A.). - An improvement in growth and egg production in chickens feed brewers dried grains. Feedstuffs, 1962, 34 (15) : $50,81,82$.

DE CLERCK. - Cours de Brasserie. Louvain Vol. I. PP. 478-481.

BAYLE, SYKES. - The Capacity of feedstuffs of tropical origin to supply nutrients for egg production. Rapport présenté au Congrès d'Aviculture de Kiev, 1966.

DERBAL (Z.) .- Précis d'Aviculture tropicale. Paris, Vigot, 1959. 